\title{
Increasing the Sensitivity of the Digital Relay Protection Against Turn-to-turn Short Circuits And Asymmetries In Wind Power Generators
}

\author{
Nedelcho Nedelchev ${ }^{1}$, and Misho Matsankov ${ }^{1}{ }^{*}$ \\ ${ }^{1}$ Technical University - Sofia, EPF-Sliven, 8800 Sliven, Bulgaria
}

\begin{abstract}
The paper describes the implementation of neural networks for increasing the sensitivity of the digital relay protection against turn-to turn short circuits in the stator winding and asymmetric modes in wind power generators. Models have been developed for monitoring two standard and two emergency mode parameters, whose deviation from the set-points is an indication of occurrence of turn-to-turn short circuits and asymmetric modes, or of combinations of these types of failures. An artificial neural network, trained by error back-propagation algorithm has been used. An experiment has been conducted to define the four criteria for studying the problem at different percentage of the short-circuit turns. A comparison between the results from both the experiment and the modeling has been made using artificial neural networks. The proposed approach, realized by means of a digital relay, allows for increasing the sensitivity of the digital relay protection against turn-to-turn short circuits and asymmetric modes for wind power generators.
\end{abstract}

\section{Introduction}

Wind power plants are mainly connected to medium voltage power grids of 6-20 kV [1]. In Bulgaria these grids operate in the three existing regimes of the star centers: isolated, grounded through an extinguishing reactor, or grounded through active resistance [2]. Only the nearby located wind power generators are connected to low-voltage $(380 \mathrm{~V})$ electric grids.

The most common failures in wind generators (WG), which are accompanied by turn-to-turn short circuits (TTSC) for stator winding generators with no parallel branches in the star center, are:

- external three-phase short circuits (SC);

- asymmetric short circuits: two-phase SC inside or outside the generator, single-phase ground connections (GC);

- disbalance current of out-of-tap generator terminals with interrupted phase of the collector feeder or in the system;

- double GC - one in the generator and the other outside in the generator in the system.

In the event of a single-phase ground connection at long-range currents in a WG, currents of ground connection over $5 \mathrm{~A}$ flow in the fault location. These currents cause significant local heating, charring and destruction of the insulation, which may result in conversion of the single-phase GC on the housing of the generator into a turn-to-turn short circuit and failure of one or two phases, located in one channel of the electric machine [3].

The turn-to-turn short circuits encompass one or several turns from one phase. They occur rarely unless they are due to insulation wear. As a consequence of the turn-to-turn short circuits, the following problems appear: ground connections on the housing, local overheating of the insulation, defects in the stator steel, and breakage of the stator phase toward the housing.

The internal short circuits can be either inter-phase short circuits with the same number of short-circuit turns for each phase (symmetric short-circuits) or asymmetric short circuits with uneven number of short circuits for each phase. The short circuits in all or part of the turns in one or two phases with short-circuit turns belong to the second group.

In case of internal short circuits, phase winding contours may occur with two current-generating branches and directional currents in the contours of the short-circuit turns in one channel. These currents have a different direction, resulting in a change in the mutual inductance of the windings [3].

Wind power plants are protected by multifunctional protection against all types of failures: phase minimum voltage protection; protection responsive to the direction of power; minimum voltage protection for lowering the linear voltage; reverse protection; asymmetry of the stator phase currents; reverse alternation of the phases; current protection without delay; current protection with depending on the current delay; maximum voltage protection; maximum current protection; directional

\footnotetext{
Corresponding author: misho.ivanov@abv.bg
} 
maximum current protection with zero sequence; protection from voltage asymmetries; protection from frequency ups and downs etc.

The current protection systems without delay (instantaneous overcurrent relay) and the maximum current protection systems with or without current delays have an operating zone, which encompasses only 65$70 \%$ of the winding. These types of protection are ineffective for limiting the volume and the degree of a failure. They only react to relatively big short circuit currents, and remain insensitive to the turn-to-turn short circuits. The emergency mode current for the turn-toturn short circuit is within 0,05 to $79 \%$ from the rated current of the generator $[4,5]$.

The applied digital protection of $\mathrm{WG}$ in some regions of Bulgaria use terminals where there is no protection against TTSC and asynchronous run [4].

Other related work demonstrates deployment of 5 $\mathrm{kW}$ wind turbines [6], others analyze performance charactetistics of wind turbines [7, 8] and [9] propose power tracking control for wind turbines.

The aim of this study is to develop models, using feedforward neural networks, while monitoring a number of parameters, by means of which turn-to-turn short circuits in the stator windings of the wind generators and asymmetric modes can be identified, as well as their combined manifestation.

\section{Criteria for identification of turn-to- turn short circuits in the stator windings of wind generators}

The turn-to-turn short circuits occur in the coiled winding of the stator. If they cause a misbalance, the action of the transversal differential protection is sufficient, as it will turn the wind generator off. When these short circuits do not cause any misbalance, which, in turn, can trigger the action of the protection, then the damage will continue to develop until it causes a breakage in the insulation of the housing or a more severe accident. Such short circuits are often nonrecognizable and the damages are considered to be due to the breakage in the insulation of the housing. Then, faulty remedial actions are identified $[4,5]$.

The mode parameters whose deviation from the setpoints are an indication of the occurrence of turn-to-turn short circuits and/or asymmetric modes in the WG are:

$\mathrm{P}$ - the active power;

$\mathrm{Q}$ - the reactive power;

$\Delta \mathrm{I}$ - the misbalance current;

$\Delta \varphi$ - the phase shift angle between the vectors of the phase currents.

Justification of the selected criteria:

In the event of a short circuit in some of the stator windings, as well as, when there is a double ground connection on the housing, a rise in the temperature in the winding is observed, accompanied by a loud buzz. A phase break or longitudinal asymmetry in the network causes a disruption of the generator operating mode. The generator decreases its rotational frequency, as well as, its power, which is accompanied by a buzz. Therefore the first parameter to be monitored is the power of the generator.

Practice shows that the reduction by $10 \%$ of the number of turns in the stator (availability of short-circuit turns) leads to increasing by $20 \%$ of the consumed reactive power [5]. Therefore the second criterion to be monitored in order to identify turn-to-turn short circuits in the stator winding is the reactive power.

The occurrence of turn-to-turn short circuits in wind generators causes electrical asymmetry in the stator windings, during which the main features of the wind generator operation are:

- disturbance of the phase current symmetry;

- change in the phase shift angle between the vectors of the phase currents;

- uneven heating of the stator windings;

- torque reduction;

- vibration and high noise level.

In asymmetric mode between the reverse sequence currents $I_{2}$ and their difference $\Delta \mathrm{I}$ in the phases, the following inequality is valid:

$$
I_{2} \leq 0.7 \Delta I,
$$

where

$$
\Delta I=\left|I_{\max }\right|-\left|I_{\min }\right|
$$

where $\left|I_{\max }\right|,\left|I_{\min }\right|$ are respectively the modules of the maximum and minimum current values in the stator phases.

The phase shift between the currents is expressed by

$$
\Delta \varphi=\varphi_{\max }-\varphi_{\min }
$$

where $\varphi_{\max }, \varphi_{\min }$ are correspondingly the maximum and minimum values of the phase shift angles between the currents in the phases of the wind generator.

Therefore the third and the fourth parameters to be monitored for identification of turn-to-turn short circuits are respectively the misbalance current $\Delta \mathrm{I}$ and the phase shift angle $\Delta \varphi$ between the vectors of the phase currents.

The four selected mode parameters are actually the four criteria, which are the input variables for building up the neural network, intended to look for turn-to-turn short circuits and asymmetric modes.

\section{Feedforward neural networks}

The main advantages of the artificial neural networks, making them applicable for research purposes are the following:

- they do not require pre-defined mathematical models;

- different types of data structures can be identified with their help;

- they have the capability of learning from examples and from data (learning through experience);

- they allow different types of complex functional dependencies to be "smoothed out", which makes them universal approximators [10-12]. 
There are different types of artificial neural networks, but the most appropriate for the purpose of the study is the forward propagation network (feedforward), which is a system, which consists of multiple neurons interconnected and organized in layers. The neural networks of this type have one input layer, one or more hidden layers and one output layer (Figure 1). The neurons of each layer are connected to the others by their weights or weight coefficients, which play the role of a "repository" of the knowledge gained during the network training. The flow of information in a feedforward network move from the input to the output layer. The role of each neuron is to process and transmit the information received from the input to the output elements.

The most important features of the neural networks are: their ability to learn and their ability to generalize. The neural networks require that the data are appropriate for training. The training of the system is realized by changing the values of the weight coefficients. The coefficients change according to the chosen training algorithm. Once the network is "trained", it must be "fed" with new data, not used during its training so far. This is the way to test the neural network in order to determine whether it is able to forecast unknown outputs or whether it is able to generalize.

\section{Compilation of a feedforward neural network for monitoring the selected parameters by which the presence of turn-to-turn short circuits is detected in the windings of the WG stator}

The structure of the neural network is determined by: the number of hidden layers; the number and type of the interconnections between them. The number of neurons in the input layer of the neural network is four. These are the four criteria, for which the values of deviation from the parameters set in a normal mode, are a signal for the occurrence of a turn-to-turn short circuit in the stator winding of the wind power generator. The criteria are: $P$ - the active power; $Q$ - the reactive power; $\Delta I-$ the misbalance current; $\Delta \varphi$ - the phase shift angle beteen the vectors of the phase currents.

The output layer is the signal for the protection to disconnect the wind generator due to the presence of a turn-to-turn short circuit.

There are no rules for determining the optimum number of hidden layers and the neurons contained in them. In order to avoid "readjusting" of the system, the neural network is chosen to have two hidden layers, where the first layer consists of 4 neurons, while the second - of 2 neurons. Therefore, the neural network has a $4 \times 4 \times 2 \times 1$ structure, which is presented in Fig. 1 for the case.

\subsection{Selection of an algorithm to train the neural network}

The error back-propagation algorithm and a given intertial parameter are used to train the FNN. The change in weights ( $\left.\Delta G_{i j(t+1)}\right)$ between the neurons $i$ and $j$ during the $t+1$ iteration is determined by:

$$
\Delta G_{i j(t+1)}=\mu \varepsilon_{i} R_{i}+\eta \Delta G_{i j(t)},
$$

where $\Delta G_{i j(t)}$ is the change in the weight between the neurons $i$ and $j$ during the $t$ iteration; $\mu$ - the learning speed; $\varepsilon_{i}$ - the error for element $j ; R_{i}$ - the result from the previous element; $\eta$ - the inertial parameter.

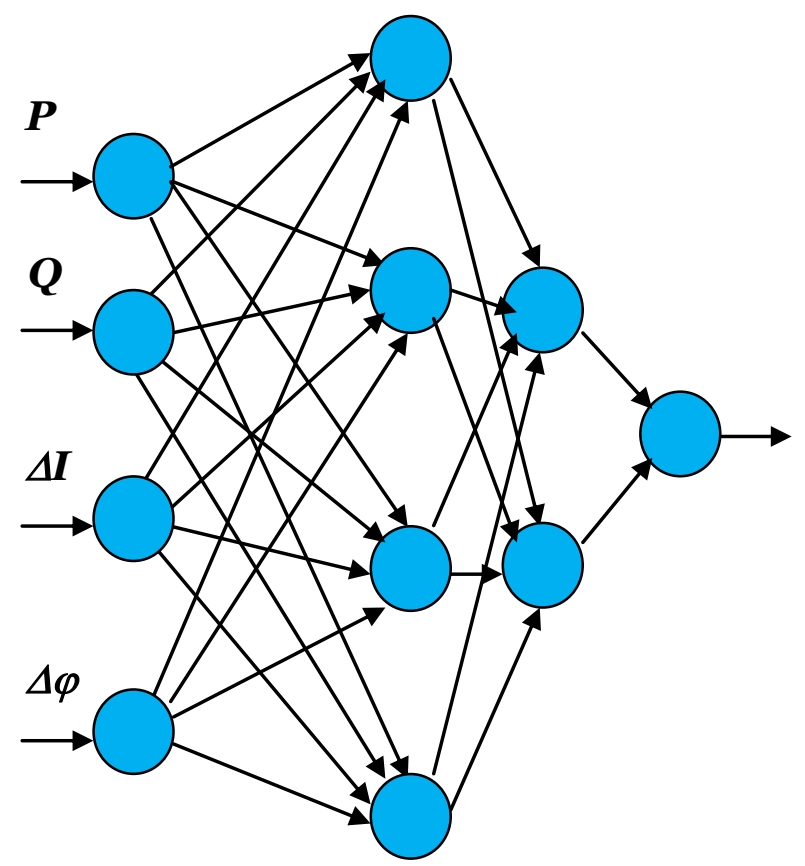

Fig. 1. FNN Structure $(4 \times 4 \times 2 \times 1)$

The inertial parameter plays the role of an "accelerator" in the training process and facilitates the establishment of different changes in the weights, combining them with the changes in the weights during the previous iteration. Most software programs use standard values for the learning coefficient 0.1 and the inertial parameter $\eta=0.9$.

\subsection{Defining the parameters of the neurons}

The chosen feedforward neural network with back propagation of the error uses an asymmetric sigmoid function. In result of multiple experiments the slope of the function (0.49) was defined, which provides the best "smoothing out" of the data.

\subsection{Selecting a model evaluation criterion}

The main purpose of the error back-propagation training algorithm is to minimize the sum of the quadratic error.

$$
\varepsilon=0,5 \sum \sum\left(H_{h i}-R_{h i}\right)^{2},
$$


where $\varepsilon$ - the sum of the quadratic error; $H_{h i}$ the desired result for the $i$-th output neuron; $R_{h i}$ - the training result for the $i$-th output neuron.

\subsection{Comparative analysis between a feedforward neural network and a real experiment}

Table 1 presents the average values of the absolute error, calculated when defining the number of the short-circuit turns in the stator winding by using a feedforward neural network and by a real experiment. The comparison is made for the biggest deviations from the calculated values both by a feedforward neural network and by measurements ( $1 \%$ difference).

Table 1. Error in defining the number of the short-circuit turns in the stator winding by using a feedforward neural network and by conducting a real experiment

\begin{tabular}{|c|c|c|}
\hline \multicolumn{2}{|c|}{$\begin{array}{c}\text { Number of short-circuit turns } \\
\text { in \%, defined }\end{array}$} \\
\cline { 1 - 2 } $\begin{array}{c}\text { experimentall } \\
\mathbf{y}\end{array}$ & \begin{tabular}{c}
\multirow{2}{*}{ Errors, \% } \\
by means of a \\
feedforward \\
neural network \\
$\mathbf{4 \times 4 \times 2 \times 1}$
\end{tabular} & \\
\hline $\mathbf{1 0}$ & 11 & \\
\hline $\mathbf{2 0}$ & 21 & 9 \\
\hline $\mathbf{3 0}$ & 31 & 5 \\
\hline
\end{tabular}

The developed FNN provides higher sensitivity of the digital relay protection against turn-to-turn short circuits.

The biggest error between the experimentally obtained number of turn-to-turn short circuits and their number, defined by the FNN, reaches up to $9 \%$ against the real $10 \%$ short-circuit turns and, correspondingly $3.33 \%$ in case of $30 \%$ short-circuit turns.

\section{Conclusions}

The neural networks are appropriate for monitoring over the selected parameters, by which the presence of turnto-turn short circuits in the stator winding of the wind generator is detected. No need of preliminary definition of a mathematical model for the use of the neural networks.

The feedforward neural networks allow for increasing the sensitivity of the digital relay protection against turn-to-turn short circuits and asymmetric modes in wind power generators.

The autors would like to thank the Research and Development Sector at the Technical University of Sofia for financial support.

\section{References}

1. Nedelcheva, S.I., Electric grids and power systems with decentralized energy generating sources, ISBN 978-619-167-348-3, Sofia, TU Sofia Publishing, Sofia, (2018).

2. Nedelchev, N.A., S.I.Nedelcheva, High voltages techniques, Part two, ISBN 978-619-167-234-9. Sofia, TU Sofia Publishing, Sofia, (2015).

3. Nedelchev, N.A., Regimes of electric power stations, Sofia, TU Sofia Publishing, (2007).

4. Nedelchev, N.A., M.I.Matzankov, S.Slavov, Protection against turn-to-turn short circuits in wind power generators, ISSN 1312-3920, Notices of TUSliven, No. 6, (2019).

5. Hongbo Qiu, Xiaobin Fan, Hua Liu, Jianqin Feng, Cunxiang Yang. Study on Operating Characteristics of Hydro Generator with Different Damper Bar Materials. Recent Patents on Engineering. Volume 12 , Issue 2, (2018).

6. Sugiyatnoa, Imam Djunaedia, Haifa Wahyub. System development and performance test of $5 \mathrm{~kW}$ wind turbine. ISSN: 2373-3594. International Journal of Smart Grid and Clean Energy, vol. 5, no. 4, October 2016: pp. 264-271.

7. Yihuai Hua, Taiyou Wanga, Hao Jina, Xianfeng Caob, Chen Zhangb. Experimental study on aerodynamic characteristics of vertical-axis wind turbine. ISSN: 2373-3594. International Journal of Smart Grid and Clean Energy, vol. 6, no. 2, April 2017: pp. 104-113.

8. Riyantoa, Nugroho Agung Pambudia, Bayu Rudiyantob, Nyenyep Sri Wardania, Rusdi Febriyantoa, Kukuh Mukti Wibowoa, Nova Dany Setyawana, Basoria, Saeid Mohammad zadeh Binac. Experimental study of the effect of blade pitch angle on the wind turbine performance at low wind speed condition. ISSN: 2373-3594. International Journal of Smart Grid and Clean Energy, vol. 8, no. 5, September 2019: pp. 627-632.

9. Soumia EL Hani, Said Guedira, Noureddine EL Alami. Maximum power tracking control wind turbine based on permanent magnet synchronous generator with complete converter. ISSN: 23154462. International Journal of Smart Grid and Clean Energy, vol. 3, no. 1, January: 2014, pp. 15-21.

10. Portogal, M ., "Neural network versus time series methods: a forecasting exercise", 14 th International symposium on forecasting, Stockholm, Sweden, (1995)

11. Remus, W., O`Connor, M., "Neural network timeseries forecasting" in Armstrong, J. (Ed.) "Principles of forecasting: a handbook for researchers and practitioners", Kluwer Academic Publishers, (2001).

12. Armstrong J.S. Principles of forecasting: A handbook for researchers and practitioners. Kluwer,Boston,MA, (2001). 